\title{
Risk-Limiting Power Grid Control with an ARMA-Based Prediction Model
}

\author{
Masahiro Ono, Ufuk Topcu, Masaki Yo, and Shuichi Adachi
}

\begin{abstract}
This paper is concerned with the risk-limiting operation of electric power grids with stochastic uncertainties due to, for example, demand and integration of renewable generation. The main contribution is incorporating autoregressive-moving-average (ARMA) type prediction models for the underlying uncertainties into chance-constrained, finitehorizon optimal control. This uncertainty model leads to a more (compared to existing work in literature) careful treatment of correlation in time which is significant especially in renewable generation yet has attracted limited attention. The paper first discusses how the resulting chance-constrained optimization problems can be solved computationally and demonstrates the effects of the use of the proposed prediction models through simulation-based case studies with realistic data.
\end{abstract}

\section{INTRODUCTION}

The increasing uncertainties due to integration of generation from renewable sources in the electric power grid necessitate new reliability metrics and new methods to assess the operation of the grid against these metrics. We consider the risk of not meeting certain constraints, e.g., supply-demand balance, as the reliability criterion, i.e., risklimiting operation or dispatch. This risk is induced from the stochasticity in renewable generation and demand. Prior work, including but not limited to [1], [2], [3], [4], on risk-limiting operation relied on simple statistical models of uncertainty and did not provide any constructive procedure to account for the effects of correlation over time. On the other hand, the outputs of the renewable generators (e.g., wind turbines) may feature significant correlation over time violating an essential assumption in this earlier work. For example, Figure 1 shows the power spectrum density of wind generation from a wind farm in Southern California in July, 2006. ${ }^{1}$ Clearly the spectral density is not uniform implying correlation over time. In other words, wind generation cannot be modeled as white noise. In addition to the recent interest in risk-limiting dispatch with no systematic treatment of correlation over time, reference [5] takes a step toward modeling correlation through a Markov chain. This approach can capture time correlation in wind generation; however, it requires to discretize power output from wind turbines into a finite number of levels.

Masahiro Ono (ono@jpl.nasa.gov) was with Keio University, Japan and JST, CREST, Japan when this research was conducted. He is currently with the Jet Propulsion Laboratory, California Institute of Technology. Ufuk Topcu (utopculseas.upenn.edu) is with the Department of Electrical and Systems Engineering at the University of Pennsylvania. Masaki Yo (masakiyo@a3.keio.jp) is with Keio University, Japan and JST, CREST, Japan. Shuichi Adachi is with Keio University, Japan.

${ }^{1}$ The data taken from by the National Renewable Energy Laboratory (NREL).

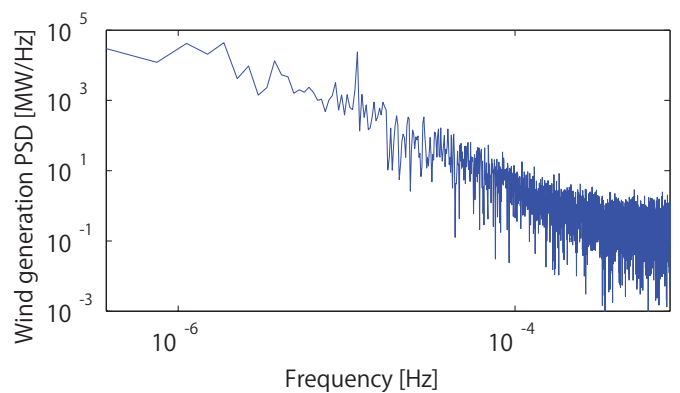

Fig. 1. Power spectrum density of wind generation at from a wind farm at Southern California in July, 2006.

On the other hand, in modeling community there is a significant body of work on the modeling of uncertain wind and demand [6]. In particular, it has been shown that an autoregressive-moving-average (ARMA) model is an effective tool to predict wind generation [7], [8] as well as electricity demand [9], [10], [11]. Moreover, unlike the Markov-chain model [5], the ARMA-based prediction models do not require discretization and can potentially capture correlation between distant time steps. However, these fruitful results have not been fully utilized in a control context.

Our work - to the best of our knowledge - takes a first step toward an integration of ARMA-based prediction models into chance-constrained model predictive control (CCMPC) while keeping an eye on the computational tractability (through modeling). We employ an ARMA model because it is simple enough to be handled by CCMPC tractably yet expressive enough to represent the behavior of wind power. More specifically, an ARMA model describes an autocorrelated stochastic process by a linear regression with additive Gaussian noise [12]. From the ARMA model we obtain a probability distribution of future wind generation and demand conditioned on the current and past observations, and use the probability distribution to evaluate the probability of constraint violations. The main contribution of the paper is this integration of the ARMA-based model for renewable generation into risk-limiting operation, which have been investigated by modeling and control communities, respectively, though in isolation from each other.

We discuss how the resulting optimization problems can be solved computationally (particularly how the chance constraints can be handled) and demonstrate the effects of the use of the proposed prediction models through simulationbased case studies with real (or relatively realistic) data. The 
main observations include the following: Without an adequate prediction model of wind and demand, increasing wind penetration (i.e., increasing uncertainties) requires a higher reserve capacity, e.g., typically supplied by conventional generation. The proposed risk-limiting control methodology with integrated ARMA-based uncertainty models yields a reduction in such reserve capacity compared to methods based on simplistic models of uncertainty. The benefits become more significant with increasing uncertainty due to, e.g., increasing wind generation penetration levels.

\section{OVERVIEW: RISK-LIMITING GRID CONTROL}

The method proposed in this paper can be considered as a wrapper that is implemented on top of an existing linear chance-constrained model predictive control (CCMPC) method and utilizes an ARMA-based prediction of stochastic processes, such as wind generation and demand. We present the method in a general way so that it can be applied to a wide variety of risk-limiting control problems. To this end, we first present a generalized form of a CCMPC problem in Section II-A. Then we apply it to simple grid control problem in Section II-B.

\section{A. General formulation of CCMPC}

We consider linear dynamics driven by stochastic disturbance input $w$ and represented by the general state-space model

$$
\begin{aligned}
x_{k+1} & =A x_{k}+B u_{k}+E w_{k}, \\
y_{k} & =C x_{k}+D u_{k}+F w_{k},
\end{aligned}
$$

where $x_{k}, u_{k}$, and $w_{k}$ are the state vector, control vector, and disturbance at time $k$, respectively.

Constraints can be represented in a general form, by proper choice of $C, D$, and $F$, as

$$
y_{k} \leq c_{k},
$$

where $c_{k}$ is a constant. Since there is uncertainty in $x_{k}$, robustly satisfying the above constraint often results in a conservative solution; when the probability distribution of $x_{k}$ is not bounded, it is typically impossible to robustly satisfy the constraint. In the CCMPC framework the deterministic constraint is replaced by a chance constraint:

$$
\operatorname{Pr}\left[y_{k} \leq c_{k}\right] \geq 1-\epsilon,
$$

where $0<\epsilon<1$ is a user-specified risk bound. We also consider additional, explicit control constraints encoded by $u_{k} \in \mathcal{U}$ at each $k$.

At time step $t$, CCMPC solves the following chanceconstrained finite-horizon optimal control (CCFHOC) problem with a given cost function $J$.

\section{Problem 1: CCFHOC}

$$
\begin{aligned}
\min & J\left(x_{t+1}, \ldots, x_{t+T}, u_{t}, \ldots, u_{t+T-1}\right) \\
\text { over } & u_{t}, \ldots, u_{t+T-1} \in \mathcal{U} \\
\text { subject to } & (1),(2), \text { and }(3), k=t, \ldots, t+T-1 .
\end{aligned}
$$

Note that $J$ can be a function of random variables $x$, such as its expectation. In many cases, the cost function is simply a function of deterministic control inputs $u$.

Various methods have been proposed to solve Problem 1. Most of the solution methods fall into one of the following two categories:

1) An analytical approach, such as [13], [14], [15], which transforms the chance constraint (3) into a deterministic constraint

$$
F_{y_{k}}^{-1}(1-\epsilon) \leq c_{k}
$$

where $F_{y_{k}}^{-1}(\cdot)$ is the inverse cumulative distribution function of $y$.

2) A scenario-based method, such as [16], [17],which evaluates the chance constraint by sampling.

The first approach is computationally efficient but requires relatively strong assumptions on the probability distribution of $w_{k}$. The second approach can be applied to relatively more general classes of problems but typically leads to higher computational cost.

The CCMPC (like the regular MPC) solves Problem 1 at each time step and applies only the initial control input.

\section{B. Example: Risk-limiting power flow and dispatch}

We review the risk-limiting power flow and dispatch problem formulated by [1] and demonstrate that it can be encoded into Problem 1. Consider a power grid consisting of a set of buses, $\mathcal{N}$. The set $\mathcal{G} \subseteq \mathcal{N}$ of generation buses is connected by transmission links with a given topology to the set $\mathcal{L} \subseteq \mathcal{N}$ of load buses, i.e., $\mathcal{N}=\mathcal{G} \cap \mathcal{L}$. A generation bus is equipped with wind turbines as well as a conventional power plant which provides ancillary services. Each load bus demands an uncertain amount of power. In order to mitigate the uncertainty in demand and wind generation, each load bus is (possibly) equipped energy storage. The problem of interest is the optimal scheduling of, for example, the ancillary services, storage, and phase angle at each bus over a finite receding time horizon.

The following notation is used. The superscript $i$ represents the index of buses while the subscript $k$ represents a time step.

$g_{k}^{i}:$ wind generation at bus $i \in \mathcal{G}$

$s_{k}^{i}$ : spinning reserve (ancillary service) at bus $i \in \mathcal{G}$

$d_{k}^{i}$ : demand at bus $i \in \mathcal{L}$

$r_{k}^{i}$ : energy inflow to the storage at bus $i \in \mathcal{L}$

$l_{k}^{i}$ : energy level of the storage at bus $i \in \mathcal{L}$

$\theta_{k_{j}}^{i}:$ phase angle at bus $i \in \mathcal{N}$

$p_{k}^{i j}$ : power flow from bus $i$ to bus $j$, for $i, j \in \mathcal{N}, i \neq j$

$b^{i j}$ : susceptance of the transmission line between bus $i$ and bus $j$, for $i, j \in \mathcal{N}, i \neq j$

Here $g_{k}^{i}$ and $d_{k}^{i}$ are uncertain quantities modeled as stochastic. The decision variables include $s_{k}^{i}, r_{k}^{i}$, and $\theta_{k}^{i}$ :

With the DC power flow approximation [18], $p_{k}^{i j}$ is a linear function of phase angles

$$
p_{k}^{i j}=b^{i j}\left(\theta_{k}^{i}-\theta_{k}^{j}\right) .
$$


The energy level of the storage is related to the charge/discharge rate through the difference equation

$$
l_{k+1}^{i}=l_{k}^{i}+r_{k}^{i} .
$$

For all the nodes, we limit the risk of violating the following constraint in order to prevent energy shortage

$$
g_{k}^{i}+s_{k}^{i} \geq d_{k}^{i}+r_{k}^{i}+\sum_{j \neq i} p_{k}^{i j}
$$

This system is encoded into the form of (1) and (2) through the choice of

$$
\begin{aligned}
& x_{k}=\left[l_{k}^{1}, \cdots, l_{k}^{n}\right]^{T}, \\
& u_{k}=\left[s_{k}^{1}, \cdots, s_{k}^{n}, r_{k}^{1}, \cdots, r_{k}^{n}, \theta_{k}^{1}, \cdots, \theta_{k}^{n}\right]^{T} \text {, } \\
& w_{k}=\left[d_{k}^{1} \cdots d_{k}^{n}, g_{k}^{1} \cdots g_{k}^{n}\right]^{T} \text {, } \\
& A=I_{n}, \quad B=I_{n}, \quad E=O_{n}, \\
& C=O_{n}, \quad D=\left[\begin{array}{lll}
-I_{n} & I_{n} L
\end{array}\right], \quad F=\left[\begin{array}{ll}
I_{n} & -I_{n}
\end{array}\right], \\
& L=\left[\begin{array}{cccc}
\sum_{j \neq 1} b^{1 j} & -b^{12} & \cdots & -b^{1 n} \\
-b^{21} & \sum_{j \neq 2} b^{2 j} & \cdots & -b^{2 n} \\
\vdots & & \ddots & \vdots \\
-b^{n 1} & \ldots & -b^{n(n-1)} & \sum_{j \neq n} b^{n j}
\end{array}\right],
\end{aligned}
$$

where $I_{n}$ and $O_{n}$ are the $n$-by- $n$ identity and zero matrices, respectively.

Furthermore, we impose upper and lower bounds on $s_{k}^{i}$, $r_{k}^{i}, l_{k}^{i}, \theta_{k}^{i}$, and $p_{k}^{i j}$. The bounds are denoted by the subscripts "min" and "max on the respective variables. We assume that $s_{k, \text { min }}^{i}$ and $l_{k, \text { min }}^{i}$ are zero for all $k$. These bounds are encoded into the feasible control set $\mathcal{U}$.

In our previous work [1], the wind generation $g_{k}^{i}$ and demand $d_{k}^{i}$ are modeled as a white Gaussian noise. Our proposed approach handles $g_{k}^{i}$ and $d_{k}^{i}$ as a colored Gaussian noise, meaning that their probability distributions are autocorrelated. In Section IV-B we demonstrate that the proposed approach significantly reduces the cost of the risk-limiting power flow and dispatch control.

\section{METHOD}

The proposed approach consists of off-line and on-line parts, as illustrated in Figure 2. An ARMA model is identified off-line using past data. This process is described in detail in Section III-A. The on-line part, which we call $C C M P C-A R M A$, consists of the ARMA model and a chance-constrained, model predictive controller. CCMPCARMA takes as an input the observed sequence of uncertain processes, such as wind generation and demand, up to the present time step. From this input the ARMA model predicts the probability distribution of the uncertain process over the current prediction horizon, as discussed in Section IIIB. Then the controller uses this probability distribution to generate a control sequence that satisfies the probabilistic constraint by solving the chance-constrained finite-horizon optimal control problem formulated in Section III-C. The on-line part is summarized in Section III-D.

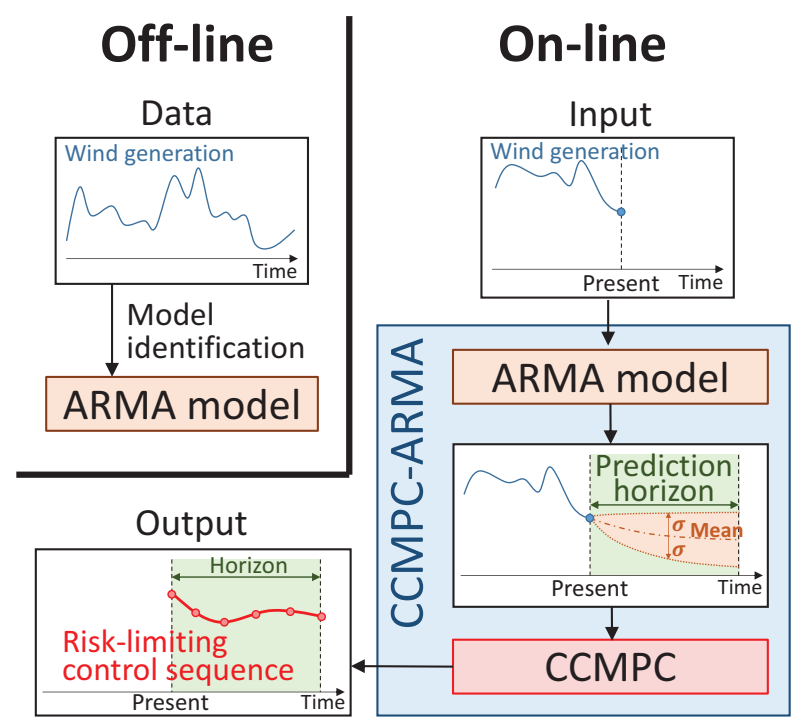

Fig. 2. Illustration of the proposed approach. An ARMA model is identified off-line using past data of uncertain process, such as wind generation and demand. During the on-line control, the ARMA model takes as an input the observed sequence of the uncertain process up to the present time step and outputs the probability distribution of the process over the current prediction horizon. Then a CCMPC controller uses this probability distribution to generate a risk-limiting control sequence.

\section{A. Construction of an ARMA model and preprocessing of data}

For now we assume $w_{k}$ to be a scalar for simplicity, while an extension to multi-dimensional case is straightforward.

An ARMA model represents a stochastic process in terms of two polynomials, one for the auto-regression (AR) and the second for the moving average (MA) [12]. More specifically, an ARMA model with $p$ autoregressive terms and $q$ movingaverage terms, denoted by $\operatorname{ARMA}(p, q)$, is

$$
w_{k}=\sum_{i=1}^{p} a_{i} w_{k-i}+\sum_{i=1}^{q} b_{i} v_{k-i}+v_{k}
$$

where $v_{k}$ is a white noise (i.e., $v_{k}$ is an independent and identically distributed random variable). An ARMA model can be viewed as a whitening filter that represents an autocorrelated (i.e., colored) process, $w_{k}$, by a a white noise $v_{k}$ [19]. It is often assumed that $v_{k}$ has a zero-mean Gaussian distribution $N\left(0, \sigma^{2}\right)$ with variance $\sigma^{2}$. In such a case, $w_{k}$ also has a Gaussian distribution, but its mean and variance are time-varying, and the distributions are autocorrelated. The maximum-likelihood estimation of the parameters, $a_{i}$, $b_{i}$, and $\sigma^{2}$, can be obtained by iterative methods such as the iterative Prony's method [20]. The order of an ARMA model is chosen to minimize a model selection criterion such as the Akaike's information criterion and the Bayesian information criterion [12].

Of our particular focus is an $\operatorname{ARMA}(p, p-1)$ model, which can be transformed into the following observable canonical 
form

$$
\begin{aligned}
z_{k+1} & =P z_{k}+Q v_{k}, \\
w_{k} & =R z_{k},
\end{aligned}
$$

where

$$
\begin{aligned}
P & =\left[\begin{array}{ccccc}
a_{1} & 1 & 0 & \cdots & 0 \\
a_{2} & 0 & 1 & & 0 \\
\vdots & \vdots & & \ddots & \\
a_{p-1} & 0 & 0 & & 1 \\
a_{p} & 0 & 0 & \cdots & 0
\end{array}\right], \quad Q=\left[\begin{array}{c}
1 \\
b_{1} \\
\vdots \\
b_{p-2} \\
b_{p-1}
\end{array}\right], \\
R & =\left[\begin{array}{llll}
1 & 0 & \cdots & 0
\end{array}\right] .
\end{aligned}
$$

The state vector $z_{k}$ is

$$
\begin{aligned}
z_{k} & =\left[w_{k}, z_{2, k}, \cdots, z_{m, k}\right]^{T} \text { with } \\
z_{j, k} & =\sum_{i=j}^{p} a_{i} w_{k+j-i-1}+\sum_{i=j-1}^{p-1} b_{i} v_{k+j-i-1} .
\end{aligned}
$$

This state-space representation of an ARMA model is integrated into a linear CCMPC in the next subsection.

It is often assumed that $v_{k}$ is a zero-mean noise. In such a case, a stationary process always results in a stable ARMA model where the mean of the stationary distribution of $w_{k}$ is zero. Therefore, before constructing an ARMA model of wind generation, we subtract the mean from the given data. For example, Figure 3-(a) shows a profile of wind generation over a month, which is used for the simulation in Section IV-B. The mean generation over the month, $12.57 \mathrm{MW}$, is subtracted from the data before computing the ARMA parameters. The mean is added back when making a prediction.

Similarly, ARMA models can be used to make a prediction of demand. Unlike wind generation, energy demand has an obvious daily pattern, as shown in Figure 3-(a). Therefore, we perform a different preprocessing for demand data. We obtain a mean at every time over the day from the given data, and subtract the circadian mean from the data. For example, in Figure 3-(b), the circadian mean is shown by the dotdashed line. As in wind generation, the circadian mean is added back when making a prediction.

\section{B. On-line prediction by the ARMA Model}

The ARMA model constructed in the previous subsection is used to make a prediction on-line. Here, we mean by prediction a set of parameters that specifies the probability distributions of $w_{k}$, such as mean and standard deviation.

Let $t$ be the present time step. Roughly speaking, the prediction can be made by recursively applying (8) and (9) starting from $k=t$. In order to do so, we first need to construct the initial state of the prediction model, $z_{t}$, using (13) and (14). This requires the values of $w_{t}, w_{t-1}, w_{t-2}, \cdots$, as well as $v_{t}, v_{t-1}, v_{t-2} \cdots$. We assume that $w_{t}, w_{t-1}, w_{t-2} \cdots$ have been observed at time step $t$, which simply means that, for example, the data of the current and past wind

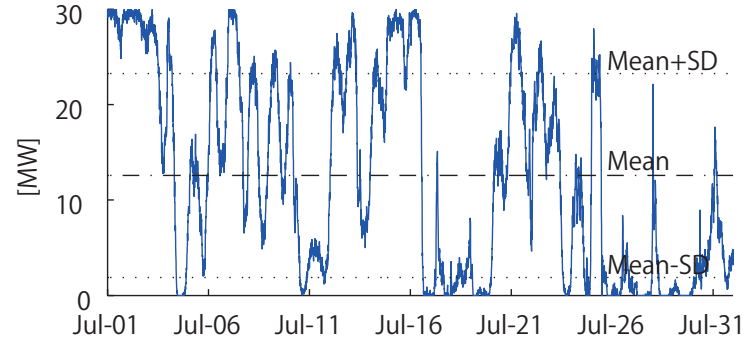

(a) Wind generation in July, 2006 at a wind farm in Southern California. Data from the National Renewable Energy Laboratory (NREL) dataset.

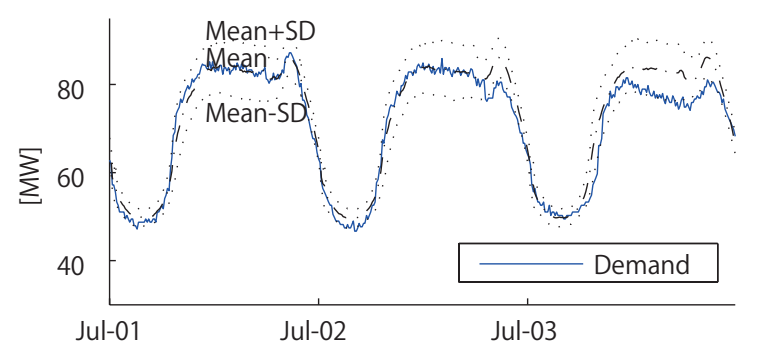

(b) Electricity demand from a typical feeder over three days in July, 2010 in the service area of Southern California Edison. The dot-dashed line represents the circadian mean of the demand.

Fig. 3. Typical profiles of wind generation and electricity demand. The one-sigma (standard deviation) interval around the mean is shown by the two dotted lines.

generation is available. However, the noise, $v_{t}$, is not directly observable. If we assume that $v_{t-1}, v_{t-2} \cdots v_{t-q}$ are available, then $v_{t}$ can be determined from the observations of $w_{t}, w_{t-1}, \cdots w_{t-p}$ by using (7) as

$$
v_{t}=w_{t}-\sum_{i=1}^{p} a_{i} w_{t-i}-\sum_{i=1}^{q} b_{i} v_{t-i} .
$$

In order to initiate this recursion, the first $q$ steps of $v_{t}$ is set to its mean value. Then, the present value of $v_{t}$ is updated on-line at each time step using (15).

Let $\bar{v}$ and $\sigma_{v}^{2}$ be the mean and variance of $v$. Then, the mean $\bar{w}_{t+k}$ and variance $\Sigma_{t+k}$ of $w_{t+k}$ are given by

$$
\begin{aligned}
\bar{w}_{t+k} & =R P^{k} z_{0}+\sum_{i=1}^{k} R P^{k-1} Q \bar{v} \text { and } \\
\Sigma_{w_{t+k}} & =\sum_{i=1}^{k} R P^{k-1} Q \sigma_{v}^{2}\left(R P^{k-1} Q\right)^{T} .
\end{aligned}
$$

C. Formulation of the chance-constrained finite-horizon optimal control problem with ARMA

We next integrate the ARMA model into Problem 1 and obtain a new formulation of the chance-constrained finitehorizon optimal control problem, which is solved at each time step in order to obtain the optimal control sequence 
over a prediction horizon. Consider the extended system

$$
\begin{aligned}
{\left[\begin{array}{l}
x_{k+1} \\
z_{k+1}
\end{array}\right] } & =A_{e}\left[\begin{array}{l}
x_{k} \\
z_{k}
\end{array}\right]+B_{e} u_{t}+E_{e} v_{t}, \\
y_{k} & =C_{e}\left[\begin{array}{l}
x_{k} \\
z_{k}
\end{array}\right]+D u_{t},
\end{aligned}
$$

where

$$
\begin{aligned}
& A_{e}=\left[\begin{array}{cc}
A & E R \\
0 & P
\end{array}\right], \quad B_{e}=\left[\begin{array}{c}
B \\
0
\end{array}\right], \\
& E_{e}=\left[\begin{array}{c}
0 \\
Q
\end{array}\right], \quad C_{e}=\left[\begin{array}{cc}
C & F R
\end{array}\right] .
\end{aligned}
$$

Then we formulate the following chance-constrained finite-horizon optimal control problem.

\section{Problem 2: CCFHOC-ARMA}

$$
\begin{array}{ll}
\min & J\left(x_{t+1}, \ldots, x_{t+T}, u_{t}, \ldots, u_{t+T-1}\right) \\
\text { over } & u_{t}, \ldots, u_{t+T-1} \in \mathcal{U}
\end{array}
$$

subject to $(18),(19)$, and $(3), k=t, \ldots, t+T-1$.

Compare (18) and (19) with (1) and (2) and notice that they have the same form. Therefore, Problem 2 can be solved by existing CCMPC approaches that can solve Problem 1 , such as the analytical and scenario-based approaches discussed in Section II-A.

For reader's convenience, we derive the mean and covariance matrix of $y_{t+k}$ below. Here we assume that the white noise $v$ is a vector, whose mean and covariance matrix are represented by $\bar{v}$ and $\Sigma_{v}$. We denote by $\bar{x}_{t}$ and $\Sigma_{x_{t}}$ the mean and covariance of the initial state $x_{t}$. Then,

$$
\begin{aligned}
\bar{y}_{k} & =C_{e} A_{e}^{k}\left[\begin{array}{c}
x_{t} \\
z_{t}
\end{array}\right]+\sum_{i=0}^{k-1} C_{e} A_{e}^{i}\left(B_{e} u_{t+i}+E_{e} \bar{v}\right)+D u_{t+k}, \\
\Sigma_{y_{k}} & =\sum_{i=0}^{k-1} C_{e} A_{e}^{i} E_{e} \Sigma_{v}\left(C_{e} A_{e}^{i} E_{e}\right)^{T}+C_{e} A_{e}^{k} \Sigma_{x_{t}}\left(C_{e} A_{e}^{k}\right)^{T} .
\end{aligned}
$$

In a special case where $\bar{v}$ follows a Gaussian distribution, the probability distribution of $y_{k}$ is also Gaussian, whose mean and variance is obtained as above. Let $F_{S G}^{-1}(\cdot)$ be the inverse cumulative distribution function of the standard Gaussian distribution, $N(0,1)$. Then the chance constraint (3) is transformed into a deterministic constraint as

$$
\bar{y}_{k}+\sqrt{\operatorname{diag}\left(\Sigma_{\mathrm{y}_{\mathrm{k}}}\right)} F_{S G}^{-1}(\epsilon) \leq c_{k},
$$

where $\operatorname{diag}\left(\Sigma_{y_{k}}\right)$ is a vector of the diagonal elements of $\Sigma_{y_{k}}$ and $\sqrt{\cdot}$ is the element-wise square root.

\section{The CCMPC-ARMA method}

The proposed method, namely CCMPC-ARMA, is summarized in Algorithm 1. At time step $t$, CCMPC-ARMA solves Problem 2 and applies only the initial control input, $u_{t}$. The main difference from typical MPCs is that CCMPCARMA must keep track of the noise process in the ARMA model, $v_{t}$, as in line 5 of Algorithm 1. In Figure 2 we illustrate prediction and control steps separately for ease of explanation. In Algorithm 1, these two steps are performed in a combined manner in line 7 since the ARMA model is integrated in the extended system (18) and (19).

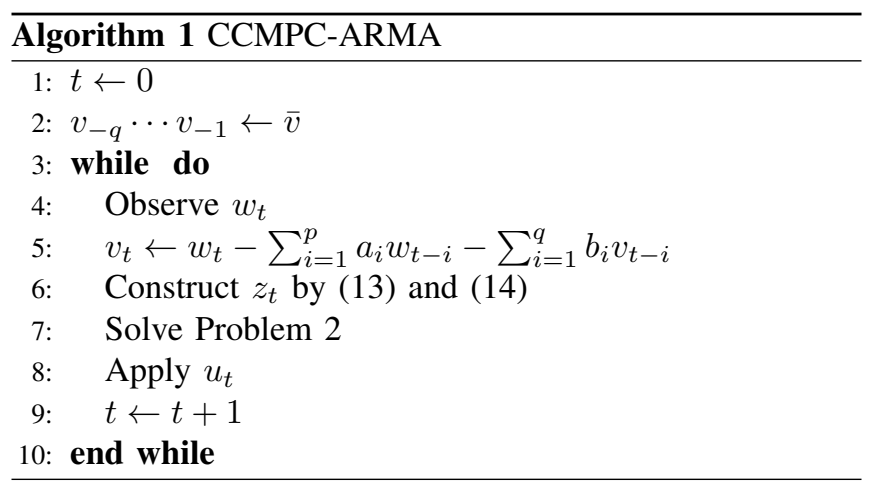

\section{Simulation Results}

\section{A. Simple dispatch problem}

We first demonstrate how the proposed method can improve the performance on a simple problem where a centralized dispatcher plans supply capacity for the next 24 hours in the presence of uncertainty in energy demand and supply. The objective is to minimize the total planned supply capacity over a day in order to minimize the cost, while making sure that the risk of being in short of supply is limited to a pre-specified bound, $\epsilon$. This risk-limiting supply capacity is computed using the proposed CCMPC-ARMA method. We compare the results with an existing approach (referred to as "CCMPC-White" hereafter) which models wind generation and demand by the sum of a circadian means and a white noise.

More specifically, we formulate the following finitehorizon optimal control problem:

$$
\min _{s_{1}, \ldots, s_{24}} \sum_{k=1}^{24} s_{k}
$$

subject to $\operatorname{Pr}\left[s_{k}+g_{k} \geq d_{k}\right] \geq 1-\epsilon, \quad k=1, \ldots, 24$,

where $g_{k}$, and $d_{k}$ are wind generation, and demand at the $k$ th time step, respectively. The planned supply capacity, denoted by $s_{k}$, represents the spinning reserve and energy generation by conventional (i.e., dispensable) power plants. Note that $g_{k}$ and $d_{k}$ are random variables predicted by the ARMA model described in Section III-A.

Observe that this problem is equivalent to Problem 1 by letting

$$
\begin{aligned}
x_{k+1} & =u_{k}=s_{k-1}, w_{k}=\left[g_{k}-\bar{g}, d_{k}-\bar{d}_{k}\right]^{T}, c=-\bar{g}+\bar{d}_{k}, \\
A & =0, B=1, B_{w}=[1,-1] .
\end{aligned}
$$

We define $w_{k}$ so that its mean is zero by subtracting the average wind generation and demand, $\bar{g}$ and $\bar{d}_{k}$. Since electricity demand has an obvious dependence on time of the day, we use circadian mean for demand, $\bar{d}_{k}$. On the other 
hand, since the dependence of wind generation on the time of the day is not as obvious as demand, we consider a constant mean $\bar{g}$ for wind. The proposed approach models the residual noise $w_{k}$ by an ARMA model while CCMPC-White models it by a white Gaussian noise.

The probability distribution of $g_{k}$ is predicted using an $\operatorname{ARMA}(3,2)$ model constructed from the real data published by California $\mathrm{ISO}^{2}$ over 93 days in December in 2010, 2011, and 2012. During these periods, approximately $2 \%$ of the total energy is supplied from wind turbines on average. In order to simulate future situations in which the renewable penetration is significantly higher than the present, we also considered cases with $20 \%, 50 \%$, and $80 \%$ wind penetrations, by magnifying the wind data. We assume that $v_{k}$ in (18) has a zero-mean Gaussian distribution with standard deviation $\sigma_{v}$. The parameters, $a_{i}, b_{i}, \sigma_{v}$, are estimated using a maximum likelihood method from the data. As for CCMPCWhite, the mean and the standard deviation at the $k$ th hour of a day are obtained statistically from the same data over 93 days.

The results are shown in Table I. We solve the above optimization problem for 93 days and the table shows the average. The cost shown in the table is the total supply capacity planned over a 3-month period, while $P_{\text {fail }}$ is the resulting probability violation at each hour, computed from the simulation over the same period. The risk bound $\epsilon$ is set to $10 \%$.

Our simulation results indicate that, with increased wind penetration, CCMPC-White requires greater amount of supply capacity because the amount of energy that can be saved by increasing wind penetration is exceeded by the extra amount of reserve capacity that is required to absorb the increased uncertainty in wind generation. Intuitively, this means that, as we introduce more wind power to a grid, we need to increase, rather than decrease, the capacity of conventional power plants in order to provide sufficient ancillary service. With such a situation, the benefit of renewable generation is significantly undermined.

The proposed approach, CCMPC-ARMA, requires smaller amount of supply capacity than CCMPC-White with all penetration levels. Most importantly, the required reserve capacity decreases as the wind penetration increases. This is because the probability distribution of future net demand (i.e., demand minus wind generation) derived from an ARMA model has significantly smaller standard deviation than a white-noise model, as shown in Figure 4. Another important feature of CCMPC-ARMA is that it uses the probability distribution of net demand conditioned on the current and past observations. For example, Figure 5 shows the mean net demands derived from an ARMA model and a white-noise model, predicted at 0:00 am on December 31. The white-noise model simply uses the same circadian mean everyday. On the other hand, an ARMA model predicts future probability distributions based on current and past few observations. Since the observed demand is lower than usual

\footnotetext{
${ }^{2} \mathrm{http}: / /$ www.caiso.com/
}

TABLE I

SimUlation RESUltS OF A SIMPLE POWER DisPATCH PROBLEM. THE TABLE SHOWS THE TOTAL REQUIRED SUPPLY CAPACITY OVER THREE MONTHS AS WELL AS THE AVERAGE PROBABILITY OF CONSTRAINT VIOLATION $\left(P_{\text {fail }}\right)$.

\begin{tabular}{|c|c|cccc|}
\hline \multicolumn{2}{|c|}{ Wind penetration } & $2 \%$ & $20 \%$ & $50 \%$ & $80 \%$ \\
\hline \hline \multirow{2}{*}{ Cost $[$ TWh $]$} & CCMPC-ARMA & 64.87 & 61.92 & 60.78 & 59.99 \\
\cline { 2 - 6 } & CCMPC-White & 65.02 & 65.72 & 70.67 & 75.90 \\
\hline \multirow{2}{*}{$P_{\text {fail }}$} & CCMPC-ARMA & $5.93 \%$ & $3.67 \%$ & $4.17 \%$ & $4.71 \%$ \\
\cline { 2 - 6 } & CCMPC-White & $4.30 \%$ & $0.32 \%$ & $0.00 \%$ & $0.00 \%$ \\
\hline
\end{tabular}

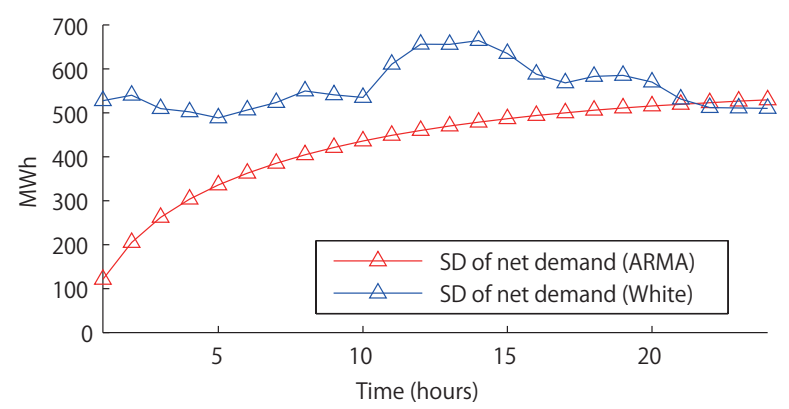

Fig. 4. Standard deviation (SD) of net demand (i.e., demand minus wind generation) on December 31, 2012

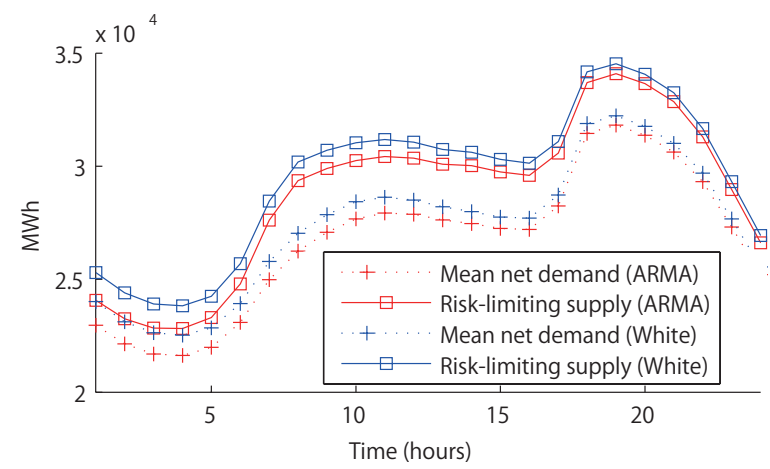

Fig. 5. Mean net demand and risk-limiting supply on December 31, 2012

over past few hours from 0:00 am on December 31, the ARMA model predicts that the mean net demand is lower than average on this day. Consequently, it requires less supply capacity and hence results in a smaller cost.

\section{B. Optimal power flow and dispatch}

Next we deploy the proposed CCMPC-ARMA method on the risk-limiting optimal power flow and dispatch problem formulated and solved by [1], which is reviewed in Section II-B. We use the IEEE 14-bus system, of which buses 1, 2, 3,6 , and 8 are generation buses. Generation profiles for the generation buses were created using data from five Southern California locations provided by NREL. At each location, the data at 10-minute intervals for five individual wind turbines during July 2006 were averaged. Load profiles were created using normalized demand data from 14 typical feeders for 
the month of July $2010 .{ }^{3}$ The data were interpolated to obtain points separated by 10-minute intervals to match the wind generation data. Samples of the data were shown in Figure 3.

In our previous work [1], uncertainty in demand and wind generation are modeled as white Gaussian noises, whose mean and standard deviation are obtained statistically from the data over 31 days of the month for each of the generation and load buses. For example, the uncertainty in the wind data at bus 1, shown in Figure 3-(a), was modeled as a white Gaussian noise with the mean being $12.57 \mathrm{MW}$ and the standard deviation being $10.68 \mathrm{MW}$. This is a quite wide distribution, whose one-sigma interval around the mean (represented by the dotted lines in Figure 3-(a)) almost covers the entire range of possible output from the wind generator. In the present work, the ARMA models are created off-line for both demand and wind generation at each bus using the same raw data. Then, during the execution of model predictive control, the mean and standard deviation of the demand and wind generation are predicted on-line using the ARMA models. The standard deviation derived from the ARMA model is significantly smaller than the white-noise model, as we show in Figure 7. Another key difference from [1] is that the previous work scheduled control actions over a single horizon while this work performs receding horizon control over a month. In this simulation study we compare the proposed approach with the receding horizon extension of [1], which we refer to as CCMPC-White for convenience.

The step cost function is a quadratic map of spinning reserve and battery charge/discharge rate

$$
J_{k}=\sum_{i \in \mathcal{G}}\left(h_{1}^{i} s_{k}^{i}+h_{2}^{i} s_{k}^{i}\right)+\sum_{i \in \mathcal{L}} h_{3}^{i}\left|r_{k}^{i}\right| .
$$

where $h_{1}^{1}=0.01, h_{1}^{2}=0.04, h_{1}^{3}=0.03, h_{1}^{6}=0.02, h_{1}^{8}=$ $0.05, h_{2}^{1}=1.5, h_{2}^{2}=1.5, h_{2}^{3}=2.0, h_{2}^{6}=2.0, h_{2}^{8}=3.0$, and $h_{3}^{i}=0.01$ for all $i \in \mathcal{L}$ in the simulations. The upper bounds of the five spinning reserves are $s_{\max }^{1}=6.0$ p.u., $s_{\max }^{2}=5.0$ p.u., $s_{\max }^{3}=4.0$ p.u., $s_{\max }^{6}=3.0$ p.u., and $s_{\max }^{8}=2.0$ p.u. We set $b_{\max }^{i}=2$ p.u., $r_{\max }^{i}=0.5$ p.u., and $r_{\min }^{i}=-0.5$ p.u. for all $i \in \mathcal{L}$. Finally, the risk bound is set to $5 \%$, i.e., $\epsilon=0.05$.

The wind generation provides $6.7 \%$ of the total energy demand. As in Section IV-A, we also conduct simulations with magnified wind supply in order to simulate scenarios with 25,50 , and $75 \%$ wind penetration. For each scenario, the CCMPC-ARMA and CCMPC-White are run over 4,000 time steps with 10-minute intervals.

Table II shows the resulting average cost per day as well as the average probability of constraint violation $\left(P_{\text {fail }}\right)$. Trends similar to those in Section IV-A are observed. Indeed, the proposed method, CCMPC-ARMA, always results in a smaller cost than CCMPC-White. Furthermore, with increasing wind penetration levels, the difference becomes more significant. The resulting probability of constraint violation

\footnotetext{
${ }^{3}$ The data is obtained through personal communication with researchers from Southern California Edison.
}

TABLE II

SiMULATION RESULTS OF A RISK-LIMITING POWER FLOW AND DISPATCH PROBLEM WITH IEEE 14 BUS SYSTEM. THE TABLE SHOWS THE AVERAGE COST PER DAY AND THE AVERAGE PROBABILITY OF CONSTRAint VIOLATION $\left(P_{\text {fail }}\right)$ OF THE PROPOSED (CCMPC-ARMA) AND EXISTING (CCMPC-WHITE) METHODS.

\begin{tabular}{|c|c|cccc|}
\hline \multicolumn{2}{|c|}{ Wind penetration } & $6.7 \%$ & $25 \%$ & $50 \%$ & $75 \%$ \\
\hline \hline \multirow{2}{*}{ Cost } & CCMPC-ARMA & 1531.6 & 1310.4 & 1059.2 & 902.5 \\
\cline { 2 - 6 } & CCMPC-White & 1694.8 & 1880.2 & 2148.1 & 2432.9 \\
\hline \multirow{2}{*}{$P_{\text {fail }}$} & CCMPC-ARMA & $6.71 \%$ & $5.95 \%$ & $5.36 \%$ & $4.71 \%$ \\
\cline { 2 - 6 } & CCMPC-White & $20.2 \%$ & $17.5 \%$ & $17.1 \%$ & $17.0 \%$ \\
\hline
\end{tabular}

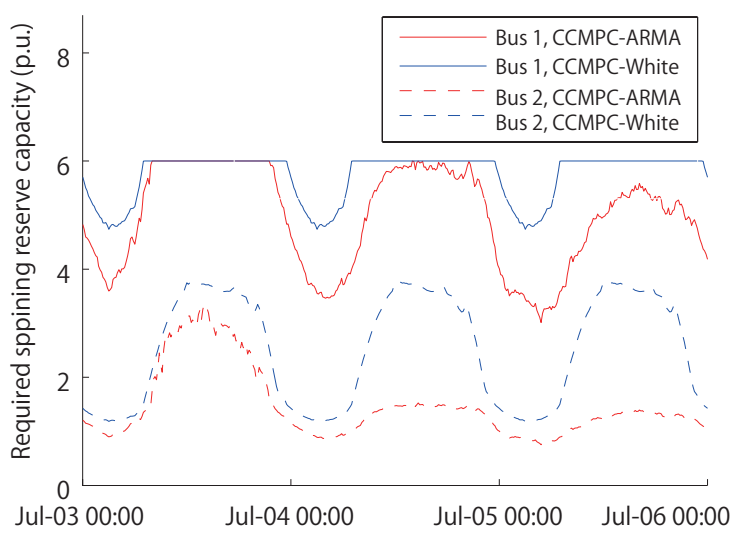

Fig. 6. Required spinning reserve capacity on buses 1 and 2 of the IEEE 14 bus system, planned by the proposed method (CCMPC-ARMA) and an existing method (CCMPC-White).

by CCMPC-ARMA is slightly above the given risk bound, $5 \%$, except for the scenario with $75 \%$ wind penetration. This is because the actual probability distribution of wind generation is not necessarily Gaussian. This issue is more remarkable in CCMPC-White since it uses a fixed probability distribution for CCMPC regardless of the current and past observations.

Figure 6 shows the profile of spinning reserve, $s_{k}^{i}$, at buses 1 and 2 over three days, generated by CCMPC-ARMA and CCMPC-White with $6.2 \%$ wind penetration. In both results the power plant at bus 1 is often utilized at the maximum output level, 6 p.u., since it has the lowest cost of generation. Observe in the figure that CCMPC-ARMA always results in smaller amounts of spinning reserve. This is the main reason of the cost reduction by CCMPC-ARMA, as shown in Table II.

CCMPC-ARMA can satisfy the chance constraint with less amounts of spinning reserve because of the improved prediction accuracy by an ARMA model. Figure 7 shows the one-sigma interval around the mean of the wind generation on bus 1 over the 12-time-step prediction horizon, generated at 0:00 on July 3. Since CCMPC-White assumes a timeinvariant probability distribution obtained off-line, its onesigma interval fails to contain the realized wind generation profile, shown in the black solid line in Figure 7 even 


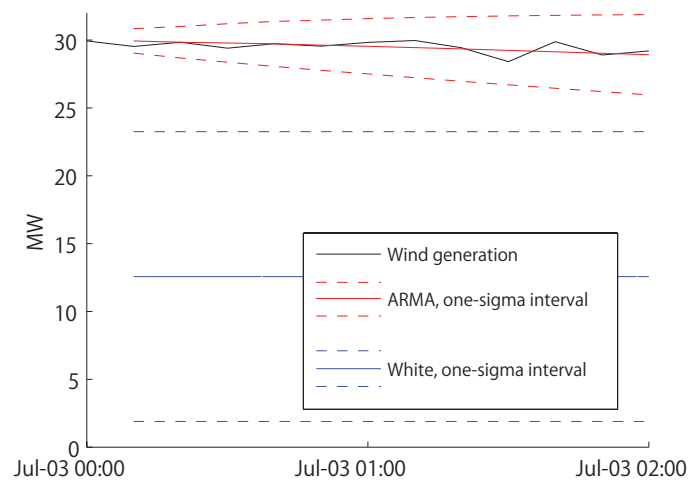

Fig. 7. Prediction of the mean and one- $\sigma$ (standard deviation) interval deviation over the prediction horizon generated by the ARMA model as well as the mean and one- $\sigma$ interval under the white noise assumption.

though having a large standard deviation. On the other hand, CCMPC-ARMA generates on-line a time-varying probability distribution conditioned on the observed data at past few time steps. Note in Figure 7 that the predicted mean of CCMPCARMA starts from the value at the present time step (i.e., 0:00 on July 3). It correctly predicts the slightly decreasing tendency of the realized wind generation profile. Also note that standard deviation of CCMPC-ARMA is significantly smaller than that of CCMPC-White, but increases over time. Intuitively, this means that the prediction of far future is more uncertain than that of near future.

In sum, the simulation results demonstrate that the proposed method can reduce the cost of risk-limiting power grid control by incorporating an ARMA model, which generates the probability distributions of wind generation and demand conditioned on the observed data.

\section{CONCLUSIONS}

We presented a risk-limiting power grid control method, namely CCMPC-ARMA, which integrates a chanceconstrained model predictive control with an ARMA-based prediction model of uncertain renewable generation and electricity demand. Unlike a white-noise model that is often assumed by existing risk-limiting power grid control methods, an ARMA-model can provide the probability distribution of future renewable generation and demand conditioned on the current and past observations, which typically has significantly smaller standard deviation. We deployed CCMPCARMA on a risk-limiting power flow and dispatch problem and empirically showed that the proposed method achieves significant saving in cost, particularly when having a high renewable penetration.

\section{ACKNOWLEDGMENT}

This work was partly supported by JST, CREST and NSF CNS award number 1312390. Any opinions, findings, and conclusions or recommendations expressed in this publication are those of the author and do not necessarily reflect the view of the sponsoring agencies. We thank Daiki Ito for his contribution in the implementation of the algorithm. We also thank A. Emma Sjödin and Dennice F. Gayme for helping us to get the data used in this work.

\section{REFERENCES}

[1] A. E. Sjödin, D. F. Gayme, and U. Topcu, "Risk-mitigated optimal power flow with high wind penetration," in Proceedings of the American Control Conference, 2012.

[2] P. Varaiya, F. Wu, and J. Bialek, "Smart operation of smart grid: Risklimiting dispatch," Proceedings of the IEEE, vol. 99, no. 1, pp. 40-57, jan. 2011.

[3] F. Bouffard and F. Galiana, "Stochastic security for operations planning with significant wind power generation," IEEE Transactions on Power Systems, vol. 23, no. 2, pp. 306-316, May.

[4] H. Ahmadi and H. Ghasemi, "Probabilistic optimal power flow incorporating wind power using point estimate methods," in Proceedings of the Conference on Environment and Electrical Engineering, 2011.

[5] M. Vrakopoulou, K. Margellos, J. Lygeros, and G. Andersson, "Probabilistic guarantees for the N-1 security of systems with wind power generation," in Proceedings of the American Control Conference, 2012.

[6] M. Lange and U. Focken, Physical Approach to Short-Term Wind Power Prediction. Springer, 2006.

[7] J. Torres, A. García, M. D. Blas, and A. D. Francisco, "Forecast of hourly average wind speed with ARMA models in Navarre (Spain)," Solar Energy, vol. 79, no. 1, pp. 65-77, 2005.

[8] L. Ling-ling, J.-H. Li, P.-J. He, and C.-S. Wang, "The use of wavelet theory and ARMA model in wind speed prediction," in Proceedings of the Conference onElectric Power Equipment - Switching Technology, 2011, pp. 395-398.

[9] S. Pappas, L. Ekonomou, D. Karamousantas, G. Chatzarakis, S. Katsikas, and P. Liatsis, "Electricity demand loads modeling using AutoRegressive Moving Average (ARMA) models," Energy, vol. 33, no. 9, pp. 1353-1360, 2008

[10] S. Pappas, L. Ekonomou, P. Karampelas, D. Karamousantas, S. Katsikas, G. Chatzarakis, and P. Skafidas, "Electricity demand load forecasting of the hellenic power system using an ARMA model,' Electric Power Systems Research, vol. 80, no. 3, pp. 256-264, 2010.

[11] Y. Ohtsuka, T. Oga, and K. Kakamu, "Forecasting electricity demand in Japan: A Bayesian spatial autoregressive ARMA approach," Computational Statistics and Data Analysis, vol. 54, no. 11, pp. 2721-2735, 2010

[12] G. E. P. Box and G. M. Jenkins, Time Series Analysis: Forecasting and Control, 3rd ed. Upper Saddle River, NJ, USA: Prentice Hall PTR, 1994.

[13] A. Charnes and W. W. Cooper, "Chance-constrained programming," Management Science, vol. 6, pp. 73-79, 1959.

[14] D. H. van Hessem, "Stochastic inequality constrained closed-loop model predictive control with application to chemical process operation," Ph.D. dissertation, Delft University of Technology, 2004.

[15] M. Ono and B. C. Williams, "Iterative risk allocation: A new approach to robust model predictive control with a joint chance constraint," in Proceedings of the IEEE Conference on Decision and Control, 2008.

[16] D. Bernardini and A. Bemporad, "Scenario-based model predictive control of stochastic constrained linear systems," in Proceedings of the IEEE Conference on Decision and Control, 2009.

[17] G. C. Calafiore, F. Dabbene, and R. Tempo, "Research on probabilistic methods for control system design," Automatica, vol. 47, no. 7, pp. $1279-1293,2011$

[18] X.-F. Wang, Y.-H. Song, and M. Irving, Modern Power Systems Analysis. Springer, 2008

[19] U. B. Desai, Modelling and Application of Stochastic Processes. Kluwer Academic Publishers, 1986.

[20] C. Therrien and C. Velasco, "An iterative prony method for ARMA signal modeling," IEEE Transactions on Signal Processing, vol. 43, no. 1, pp. 358-361, Jan. 